\title{
A Discrete-Space Train Movement Model for a High-Speed Train under Temporary Speed Restriction
}

\author{
Sihui Long $\mathbb{D},{ }^{1}$ Lingyun Meng $\mathbb{D},{ }^{2}$ Yihui Wang $\mathbb{D}^{1},{ }^{1}$ Jianrui Miao $\mathbb{D},{ }^{1}$ and Xuan $\mathrm{Li}^{2}$ \\ ${ }^{1}$ State Key Laboratory of Rail Traffic Control and Safety, Beijing Jiaotong University, No. 3 Shangyuancun, Beijing 100044, China \\ ${ }^{2}$ School of Traffic and Transportation, Beijing Jiaotong University, No. 3 Shangyuancun, Beijing 100044, China \\ Correspondence should be addressed to Jianrui Miao; jrmiao@bjtu.edu.cn
}

Received 6 January 2020; Revised 31 March 2020; Accepted 16 April 2020; Published 19 May 2020

Academic Editor: Andrés Sáez

Copyright (c) 2020 Sihui Long et al. This is an open access article distributed under the Creative Commons Attribution License, which permits unrestricted use, distribution, and reproduction in any medium, provided the original work is properly cited.

This paper constructs a discrete-space train movement model to evaluate the impact of a temporary speed restriction (TSR) for a high-speed railway train. The established model can demonstrate train movement under different TSR conditions. The proposed model can reveal whether a train is affected by the block section influenced by the TSR within a time duration. Moreover, the model can output detailed train trajectories and the minimal train running time between two adjacent stations to analyse the impact of the TSR. Based on the experimental results, we carry out a comprehensive analysis of the impact of several factors on the running time and train trajectories, including the length of the affected area (i.e., number of affected block sections), the location of the TSR, the limited speed value, and the stopping patterns of the train at two adjacent stations. The experiments show that the proposed discrete-space train movement model can be used to analyse the impact of the TSR on a high-speed railway train under various considered TSR conditions.

\section{Introduction}

The high-speed railway system is of crucial importance for the stability, sustainability, and efficiency of railway transportation systems. However, in daily railway operations, some perturbations, such as the temporary speed restriction (TSR), may influence train operations. The perturbation caused by a TSR can affect up to $85 \%$ of all events (Xu et al. [1]). In Chinese high-speed railway operations, a TSR may be applied due to bad weather, infrastructure failure, maintenance requirements, and so on.

A speed reduction caused by a TSR will cause a longer running time in the affected area and further affect train punctuality. If a TSR occurs in real operations, the train dispatchers are responsible for issuing the TSR command to train drivers and then making decisions regarding the rescheduling of the timetable (e.g., the arrival and departure times at stations, the train orders, and the train routes). The arrival and departure times are decided based on a known running time between two adjacent stations. In practice, the planned train running time between two adjacent stations is given based on a fixed maximum running speed for a line section without considering perturbations. After a TSR occurs, most dispatchers presently still make running decisions manually based on their experiences and professional expertise, typically without rigorous computations. Different TSR conditions may differ in many factors, e.g., the number of affected block sections, the location of the TSR, and the limited speed value, which makes the dispatching task more difficult. Moreover, a major train collision (i.e., the Zibo train collision), with a death toll of 72 people and 416 injuries, occurred on April 28, 2008, in China because a train was travelling much faster than the speed limit in the temporary speed restriction segment. How to avoid similar incidents is an important issue to be studied. Therefore, research on the impact of the TSR under different conditions is significant for efficient and safe train operations.

Recently, research on the TSR has received much attention. Wang and Zhang [2] analysed the methods for the transmission and execution of the TSR in different Chinese train control systems and then proposed a real-time method to transmit the TSR information between the onboard and 
ground equipment. Xu et al. [3] presented an MILP model to solve the dispatching problem under a TSR. Moreover, they analysed some parameters of speed limitations, i.e., the number of disrupted sections, the level of speed limitation, and the duration of the TSR. Long et al. [4] developed a mixed-integer linear programming model to solve the train rescheduling optimization problem by considering train control when disruptions occur during high-speed railway operations. Li et al. [5] proposed a rescheduling optimization model that considers TSR commands to assist railway dispatchers. A paper by van der Kooij et al. [6] used real train traffic data to analyse the effect of the TSR in railways. However, only three factors were included in these studies, i.e., the difference between the normal speed and reduced speed, the length of the TSR, and the length and weight of the train.

The main contributions of this paper are as follows. First, we study the impact of the TSR on a high-speed train based on a novel discrete-space train movement model. Second, the proposed model is used to determine whether a train will be affected by the block section influenced by a TSR within a time duration. The model can calculate the train trajectories in detail and the train running time between two adjacent stations under different TSR conditions. Third, we conduct a comprehensive analysis of the impact of several factors of the TSR, including the number of affected block sections, the location of the TSR, the limited speed value, and the stopping patterns of a train at two adjacent stations.

The rest of this paper is organized as follows. In Section 2 , a discrete-space train movement model is presented and a piecewise linear approach is introduced to approximate the nonlinear speed-related constraints. Section 3 illustrates the experimental settings, including the parameters of the line and train. Section 4 first evaluates the model performance and then uses 8 sets of numerical examples to simulate the effect of a TSR on a high-speed train based on the proposed model. Finally, Section 5 ends the paper with conclusions and directions for future research.

\section{Discrete-Space Train Movement Model}

The train movement model determines the speed and space profiles when a train traverses a railway line (Yang et al. [7]). Some researchers have studied train movement based on the discrete-time approach. For example, the paper by Yang et al. [7] showed that the traffic capacity of a rail line will decrease with the influence of stochastic disturbance based on a discrete-time model. Sun et al. [8] presented a new simulation approach for solving the mixed train scheduling problem on a high-speed double-track rail line based on the discrete-time movement model. However, as proposed in previous studies (Franke et al. [9], Howlett [10], Khmelnitsky [11], Liu and Golovitcher [12], and Wang [13]), it is better to choose space as an independent variable rather than time. The choice of space as the independent variable simplifies the consideration of track-related data, e.g., the speed limits and the line condition, including the curves, grades, and tunnels. Moreover, the discrete-space method has been used in many previous studies to study the train control problem (e.g., Lu et al. [14], Tan et al. [15], and Wu et al. [16]).

In this section, we propose a discrete-space train movement model under a temporary speed restriction (TSR) to calculate the train trajectory and the minimal running time between two adjacent stations. The details of the model are as follows.

The objective function is used to minimize the total running time. In addition, the riding comfort is considered, which is expressed as a function of the change in the acceleration variable $\mathrm{acc}_{i, k}$, since reducing the number of transitions and the rate of change in $\operatorname{acc}_{i, k}$ may improve passenger comfort. The objective function can be written as follows:

$$
Z=\min \sum_{i} \sum_{k} \Delta t_{i, k}+\lambda \sum_{i} \sum_{k}\left(\operatorname{acc}_{i, k+1}-\operatorname{acc}_{i, k}\right),
$$

where $Z$ is the weighted integral of the total delay times and riding comfort, $\lambda>0$ is the weight, $\Delta t_{i, k}$ is the running time of the train at segment $k$ on block section $i$, and $\operatorname{acc}_{i, k}$ is the acceleration of the train at speed point $k$ on block section $i$.

To model whether the train will be affected by the TSR area or not within a time duration, the train arrival time $a_{i}$ and departure time $d_{i}$ at each block section need to be calculated as

$$
\begin{aligned}
d_{i} & =a_{i}+\sum_{k=1}^{|K|} \Delta t_{i, k}, \quad \forall i, k=1, \ldots,|K|, \\
a_{i+1} & =d_{i}, \quad \forall i, \\
a_{1} & =T_{0},
\end{aligned}
$$

where $|K|$ is the number of segments on a block section and $T_{0}$ is the planned time at the start of the line.

The TSR-affected block sections typically have a time duration of $\left[t_{i}^{\text {sta }}, t_{i}^{\text {end }}\right]$, in which $t_{i}^{\text {sta }}$ and $t_{i}^{\text {end }}$ are the start and end times of the TSR, respectively. We use two 0-1 variables $\lambda_{1, i}$ and $\lambda_{2 . i}$ to determine whether the train has left block section $i$ before the start of the TSR and has entered block section $i$ after the end of the TSR:

$$
\begin{aligned}
\left(\lambda_{1, i}-1\right) \times M \leq d_{i}-t_{i}^{\mathrm{sta}}-\varepsilon, & \forall i \in \mathrm{TSR}, \\
\lambda_{1, i} \times M \geq d_{i}-t_{i}^{\mathrm{sta}}-\varepsilon, & \forall i \in \mathrm{TSR}, \\
\left(\lambda_{2, i}-1\right) \times M \leq t_{i}^{\mathrm{end}}-a_{i}-\varepsilon, & \forall i \in \mathrm{TSR}, \\
\lambda_{2, i} \times M \geq t_{i}^{\mathrm{end}}-a_{i}-\varepsilon, & \forall i \in \mathrm{TSR},
\end{aligned}
$$

where $M / \varepsilon$ is a sufficiently large/small positive number. Then, we consider a $0-1$ binary variable $\xi_{i}$ to satisfy the following condition: $\lambda_{1, i}=1 \cap \lambda_{2, i}=1 \Leftrightarrow \xi_{i}=1$, which determines whether the train will be in the TSR area in the time duration $\left[t_{i}^{\text {sta }}, t_{i}^{\text {end }}\right]$. The abovementioned condition can be linearized as follows:

$$
\begin{array}{rc}
-\lambda_{1, i}+\xi_{i} \leq 0, & \forall i, \\
-\lambda_{2, i}+\xi_{i} \leq 0, & \forall i, \\
\lambda_{1, i}+\lambda_{2, i}-\xi_{i} \leq 1, & \forall i .
\end{array}
$$


When the train is affected by the TSR (i.e., $\xi_{i}=1$ ), then the train speed in the TSR area $i$ cannot exceed the limited speed value $v_{i}^{\text {TSR }}$, which can be written as

$$
\left(1-\xi_{i}\right) \times v_{i}^{\max } \geq v_{i, k}-v_{i}^{T S R}, \quad \forall i, k=1, \ldots,|K| .
$$

In the equation, $v_{i}^{\max }$ is the speed limit on block section $i$. We consider a high-speed railway line at the microscopic level with block sections that connect two nodes (i.e., signals). The speed limit, track grade, curves, and tunnels of each segment at a block section are assumed to be constant. Moreover, the train is assumed to have uniformly accelerated or decelerated motion in each distance segment with a constant acceleration. Each block section is considered to be divided into ten segments. The motion of a train in each segment can be described by a discrete-space method as written in the following equation:

$$
\rho m \times \operatorname{acc}_{i, k}=\mu_{i, k}-R_{i, k}^{b}-R_{i, k}^{1}, \quad \forall i \in N, k=1, \ldots,|K| .
$$

In the equation, $\rho$ and $m$ indicate the rotating mass factor and the mass of the train, respectively; $i \in N$ denotes the set of nodes in the line; $\mu_{i, k}$ is the traction or braking force; $R_{i, k}^{b}$ denotes the basic resistance; and $R_{i, k}^{1}$ is the line resistance caused by the track grade, curves, and tunnels.

$\mu_{i, k}$ is bounded by the maximum traction force $u_{i, k}^{\max }$ and the maximum braking force $u_{i, k}^{\min }$. The maximum braking force for service braking is taken to be constant in this model to simplify the calculation (Hansen and Pachl [17] and Wang [13]). The maximum traction force is a function of the speed, which can be written as equation (8) if the train speed $v_{i, k}$ belongs to the interval $\left[v_{f, r}, v_{f, r+1}\right]$ :

$$
\begin{aligned}
& u_{i, k}^{\min } \leq u_{i, k} \leq u_{i, k}^{\max }, \quad \forall i, k=1, \ldots,|K|, \\
& u_{i, k}^{\max }=c_{1, r}+c_{2, r} v_{i, k}+c_{3, r} v_{i, k}^{2}, \quad v_{i, k} \in\left[v_{r}, v_{r+1}\right], k=1, \ldots,|K|,
\end{aligned}
$$

where $r$ is the number of switching points for the train speed in the calculation of the maximum traction force and $c_{1, r}$, $c_{2, r}, c_{3, r}, v_{r}$, and $v_{r+1}$ are determined by the train characteristics.

According to the Davis formula, the basic resistance of the train can be written as

$$
R_{i, k}^{b}=m g \times\left(A+B \times v_{i, k}+C \times v_{i, k}^{2}\right), \quad \forall i, k=1, \ldots,|K|,
$$

where $g$ is the gravitational acceleration and $A, B$, and $C$ are determined by the characteristics of the train.

The line resistance caused by the track slope, curves, and tunnels can be described as

$$
R_{i, k}^{1}=m g \times \frac{\alpha_{i}}{1000}+f_{c}\left(\operatorname{cur}_{i}\right)+f_{t}\left({ }_{i} L_{i}^{\text {tun }}\right), \quad \forall i, k=1, \ldots,|K|,
$$

where $\alpha_{i}$, $\operatorname{cur}_{i}$, and $L_{i}^{\text {tun }}$ are the track slope, curve radius, and tunnel lengths on block section $i$, respectively. The curve resistance $f_{c}\left(\right.$ cur $\left._{i}\right)$ can be described by Roeckl's formula (Wang [13]) as

$$
f_{c}\left(\operatorname{cur}_{i}\right)= \begin{cases}\frac{6.3}{\left(\operatorname{cur}_{i}-55\right)}, & \operatorname{cur}_{i} \geq 300(\text { unit : m), } \\ \frac{4.91}{\left(\operatorname{cur}_{i}-30\right)}, & \operatorname{cur}_{i}<300 \text { (unit: m). }\end{cases}
$$

The tunnel resistance $f_{t}\left({ }_{i} L_{i}^{\text {tun }}\right)$ can be given as follows:

$$
f_{t}\left({ }_{i} L_{i}^{\mathrm{tun}}\right)=0.00013 \times L_{i}^{\mathrm{tun}}, \quad \forall i .
$$

The distance and time between $v_{i, k}$ and $v_{i, k+1}$ is denoted by $\Delta L_{i, k}$ and $\Delta t_{i, k}$. The relationship between $\Delta L_{i, k}$ and $\Delta t_{i, k}$ is as follows:

$$
\Delta t_{i, k}=\frac{\Delta L_{i, k}}{\left(v_{i, k}+v_{i, k+1}\right) / 2}, \quad \forall i, k=1, \ldots,|K|,
$$

where $\Delta L_{i, k}=L_{i} /|K|$, in which $L_{i}$ is the length of block section $i$.

The train speeds at the beginning and end of the line are defined as

$$
\begin{aligned}
v_{1,1} & =v_{o}, \\
v_{|N|, 11} & =v_{e},
\end{aligned}
$$

where $|N|$ is the total number of nodes and $v_{0}$ and $v_{e}$ are the starting and ending speeds of the train, respectively.

The train speed cannot exceed the given speed limits on each block section:

$$
0 \leq v_{i, k} \leq v_{i}^{\max }, \quad \forall i, k=1, \ldots,|K| .
$$

To ensure the consistency of the train speed between two adjacent block sections, the speed of the train at the end speed points on block section $i$ equals its speed at the first speed points on block section $i+1$ :

$$
v_{i,|K|+1}=v_{i+1,1}, \quad \forall i .
$$

Finally, to linearize the nonlinear speed-related constraints (8), (9), and (13), the following mixed-integer constraints determined by using a similar piecewise linear approach as in Tan et al. [15] and Wu et al. [16] should be imposed on the model:

$$
\begin{aligned}
v_{i, k}^{\mathrm{avg}} & =\frac{v_{i, k}+v_{i, k+1}}{2}, \quad \forall i, k=1, \ldots,|K|, \\
v_{i, k}^{\mathrm{avg}} & =\sum_{x=1}^{n}\left(V_{i}^{\min }+(x-1) \times \omega\right) \times \beta_{x, i, k}, \quad \forall i, k=1, \ldots,|K|, \\
\frac{1}{v_{i, k}^{\mathrm{avg}}} & =\sum_{x=1}^{n} \frac{1}{V_{i}^{\min }+(x-1) \times \omega} \times \beta_{x, i, k}, \quad \forall i, k=1, \ldots,|K|, \\
\sum_{x=1}^{n} \beta_{x, i, k} & =1, \quad \forall i, k=1, \ldots,|K|, \\
0 & \leq \beta_{x, i, k} \leq 1, \quad \forall i, x=1, \ldots, n, k=1, \ldots,|K|,
\end{aligned}
$$


where $v_{i, k}^{\text {avg }}$ is the average speed of $v_{i, k}$ and $v_{i, k+1}, \omega$ is a small constant representing the linear sections within a valid piecewise linear range from $V_{i}^{\min }$ to $V_{i}^{\max }$, $n=\left(V_{i}^{\max }-V_{i}^{\min }\right) / \omega$, and $\beta_{x, i, k}, x=1, \ldots, n$ is a series of nonnegative variables of special ordered sets type 2 (SOS2).

Another set of SOS2 could be used to linearize $v_{i}^{2}$ of each segment, where $\vartheta_{x, i, k}$ are variables of this SOS2 in each segment:

$$
\begin{aligned}
& v_{i, k}=\sum_{x=1}^{n}\left(V_{i}^{\min }+(x-1) \times \omega\right) \times \vartheta_{x, i, k}, \quad \forall i, k=1, \ldots,|K|, \\
& v_{i, k}^{2}=\sum_{x=1}^{n}\left(V_{i}^{\min }+(x-1) \times \omega\right)^{2} \times \vartheta_{x, i, k}, \quad \forall i, k=1, \ldots,|K| .
\end{aligned}
$$

\section{Experimental Settings}

This section provides a description of the experimental settings based on a high-speed railway line with two stations, approximately 85.606 kilometres long. Figure 1 shows a detailed layout of all block sections in the line. The line is composed of 48 nodes and 47 block sections, the average length of which is $1821 \mathrm{~m}$ per block section. The detailed lengths of all block sections are illustrated in Table 1. One direction from station A to station B is considered. As illustrated in Figure 1, there are four tunnels without a limiting gradient on the line, which are located at positions [21395, 22859], [32660, 33660], [68630, 70130], and [70542, 72042] (unit: $\mathrm{m}$ ). Figure 2 shows the grade profile and the curve radius of the line. The parameters of the high-speed train are shown in Table 2. The model is solved by the CPLEX solver, version 12.3, with the default settings. The following experiments are all performed on an Intel(R) Core(TM) i7-4770 CPU with a $3.40 \mathrm{GHz}$ processor and $4 \mathrm{~GB}$ of RAM.

\section{Experimental Results and Analysis}

4.1. Experimental Results regarding the Model Performance. This section studies the solution quality and computational efficiency of the proposed model by considering different numbers of segments $|K|$ and different numbers of linear sections $n$. As discussed before, we divide each block section into $|K|$ segments to simplify the calculation of the train movement, and we use a piecewise linear approach to linearize the nonlinear speed-related constraints, in which $n$ is the total number of linear sections. In addition, the precision of the results is related to the values of parameters $|K|$ and $n$. Larger $|K|$ and $n$ will yield more precise results. However, the values of these parameters are limited by the available computing power, as well as the complexity of the decision model. In this study, we focus on the influence of the values of $|K|$ and $n$ on the running time between two stations, the problem size (i.e., the number of variables and the number of constraints), and the computational time of the proposed model. The experimental results are shown in Tables 3 and 4.

Regarding the influence of different values of $|K|$, as illustrated in Table 3 , we can see that, from $|K|=1$ to

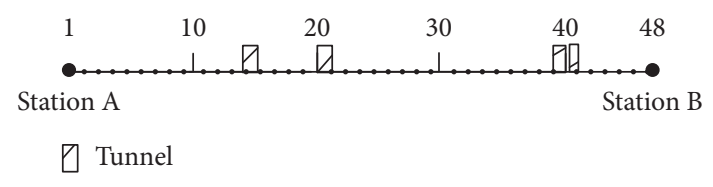

FIgURE 1: The layout of the block sections.

TABLE 1: Length of the block sections.

\begin{tabular}{lccccc}
\hline BS* $^{*}$ & $\mathrm{~L}^{* *}$ & $\mathrm{BS}$ & $\mathrm{L}$ & $\mathrm{BS}$ & $\mathrm{L}$ \\
\hline $1-2$ & 1443 & $17-18$ & 1856 & $33-34$ & 1911 \\
$2-3$ & 1406 & $18-19$ & 1913 & $34-35$ & 1859 \\
$3-4$ & 1725 & $19-20$ & 1864 & $35-36$ & 1856 \\
$4-5$ & 1795 & $20-21$ & 1911 & $36-37$ & 1863 \\
$5-6$ & 1598 & $21-22$ & 1907 & $37-38$ & 1865 \\
$6-7$ & 1350 & $22-23$ & 1914 & $38-39$ & 1914 \\
$7-8$ & 1607 & $23-24$ & 1906 & $39-40$ & 1912 \\
$8-9$ & 1919 & $24-25$ & 1912 & $40-41$ & 1914 \\
$9-10$ & 1905 & $25-26$ & 1849 & $41-42$ & 1914 \\
$10-11$ & 1879 & $26-27$ & 1863 & $42-43$ & 1914 \\
$11-12$ & 1909 & $27-28$ & 1915 & $43-44$ & 1911 \\
$12-13$ & 1911 & $28-29$ & 1914 & $44-45$ & 1915 \\
$13-14$ & 1912 & $29-30$ & 1911 & $45-46$ & 1771 \\
$14-15$ & 1896 & $30-31$ & 1880 & $46-47$ & 1644 \\
$15-16$ & 1906 & $31-32$ & 1906 & $47-48$ & 1081 \\
$16-17$ & 1866 & $32-33$ & 1914 & & \\
\hline
\end{tabular}

BS: block Section, indicated by the start node and end node of the block section. L: length of the block section, unit: $\mathrm{m}$.

$|K|=10$, the running time undergoes a notable increase since the model becomes more precise in reflecting the actual train movement. From $|K|=10$ to $|K|=20$, the value of the running time exhibits no significant change, while the computational time noticeably rises due to the introduction of many more variables and constraints. Thus, we use $|K|=10$ to obtain the comparison results by changing the value of $n$. As shown in Table 4 , the running time sees a significant increase from $n=5$ to $n=10$, while from $n=10$ to $n=20$, the increase in the running time is not significant. In Section 4.2 , further experiments are performed with $|K|=10$ and $n=10$ with some model precision and a modest computational time.

4.2. Experimental Results regarding the Effect of the Temporary Speed Restriction. In this section, we use 8 sets of numerical examples to simulate the effect of the temporary speed restriction on a high-speed train based on the proposed MILP model. We first show the train trajectories without the temporary speed restriction (TSR) in Figure 3. The running time of the case in which the train stops at both stations A and $B$ (i.e., 1354 seconds) is greater than that of the case in which the train does not stop at either station A or B (i.e., 1027 seconds) due to the time lost during train acceleration and deceleration.

In the following experiments, we use the cases without the TSR as the benchmark for the other cases with and without stops at stations to evaluate the impact of the TSR. The following sections quantify the impact of different factors using the time loss from the TSR. A time loss of Case 


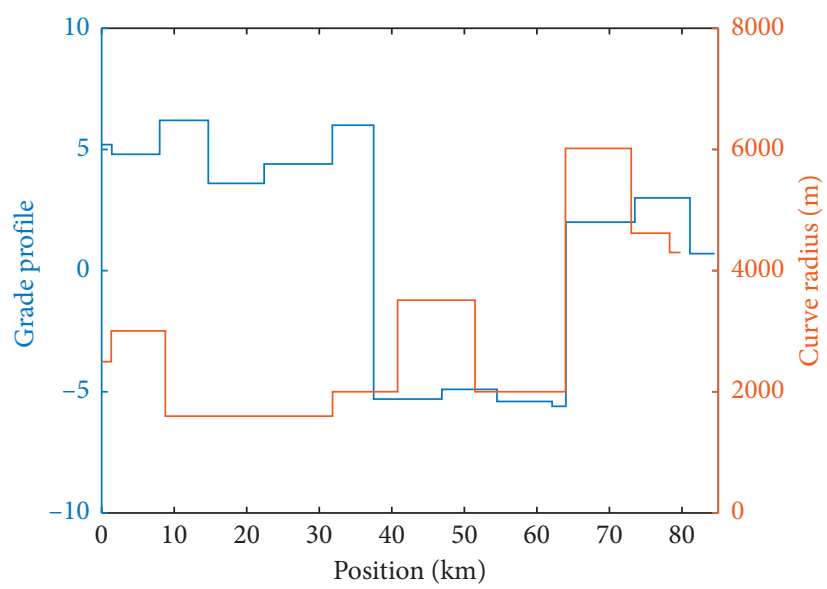

FIGURE 2: The grade profile and the curve radius of the line.

TABle 2: Parameters of a typical high-speed train.

\begin{tabular}{lccccc}
\hline $\begin{array}{l}\text { Train mass } \\
(\mathrm{kg})\end{array}$ & $\begin{array}{c}\text { Train length } \\
(\mathrm{m})\end{array}$ & $\begin{array}{c}\text { Mass factor } \\
(-)\end{array}$ & $\begin{array}{c}\text { Maximum braking } \\
\text { force }(\mathrm{N})\end{array}$ & Basic resistance $(\mathrm{N} / \mathrm{kg})$ & \multicolumn{1}{c}{$\begin{array}{c}\text { Maximum traction force } \\
(\mathrm{N})\end{array}$} \\
\hline & & & & & $v_{i, k} \in[0,36.11]:$ \\
429200 & 203.5 & 1.08 & -555000 & $A=0.55, B=0.004$, & $\begin{array}{l}c_{1}=280000, c_{2}=-245, c_{3}=0 \\
v_{i, k} \in[36.11,83.33]: \\
c_{1}=405968.3, c_{2}=-1434, c_{3}=1.693\end{array}$ \\
\hline
\end{tabular}

Table 3: Performance comparison for different numbers of segments.

\begin{tabular}{lcccc}
\hline$|K|$ & Running time $(\mathrm{sec})$ & \# of variables & \# of constraints & Computational time (sec) \\
\hline 1 & 1160.8 & 2585 & 3528 & 0.33 \\
3 & 1205.5 & 6533 & 8510 & 1.50 \\
5 & 1311.8 & 10481 & 13492 & 7.72 \\
10 & 1353.8 & 20351 & 25947 & 23.61 \\
20 & 1358.5 & 40091 & 50857 & 839.06 \\
\hline
\end{tabular}

TABle 4: Performance comparison for different $n$.

\begin{tabular}{lcccc}
\hline$n$ & Running time $(\mathrm{sec})$ & \# of variables & \# of constraints & Computational time (sec) \\
\hline 5 & 1245.9 & 15651 & 23597 & 12.83 \\
10 & 1353.8 & 20351 & 25947 & 24.91 \\
15 & 1354.5 & 25051 & 28297 & 42.05 \\
20 & 1362.1 & 29751 & 30647 & 86.73 \\
\hline
\end{tabular}

$\mathrm{x}$ is calculated by (the running time of Case $\mathrm{x}$, the running time of the case without a TSR). For each TSR case, we report the train trajectories and the time loss relative to the normal speed (i.e., cases without a TSR).

4.2.1. Impact of the Number of Affected Block Sections. This section illustrates the impact of the number of affected block sections. Without loss of generality, we use two sets of experiments with different stopping patterns to analyse the time loss and train trajectories. The time loss of each experiment is illustrated in Figure 4. Train trajectories with different stopping patterns under different numbers of affected block sections are illustrated in Figures 5 and 6. From the analysis of Figure 4, the time loss increases as the number of affected block sections increases. There is a linear trend between the numbers of affected block sections and the time loss under the TSR. However, there are different time losses for different stopping patterns, which is analysed in detail in Section 4.2.5. 


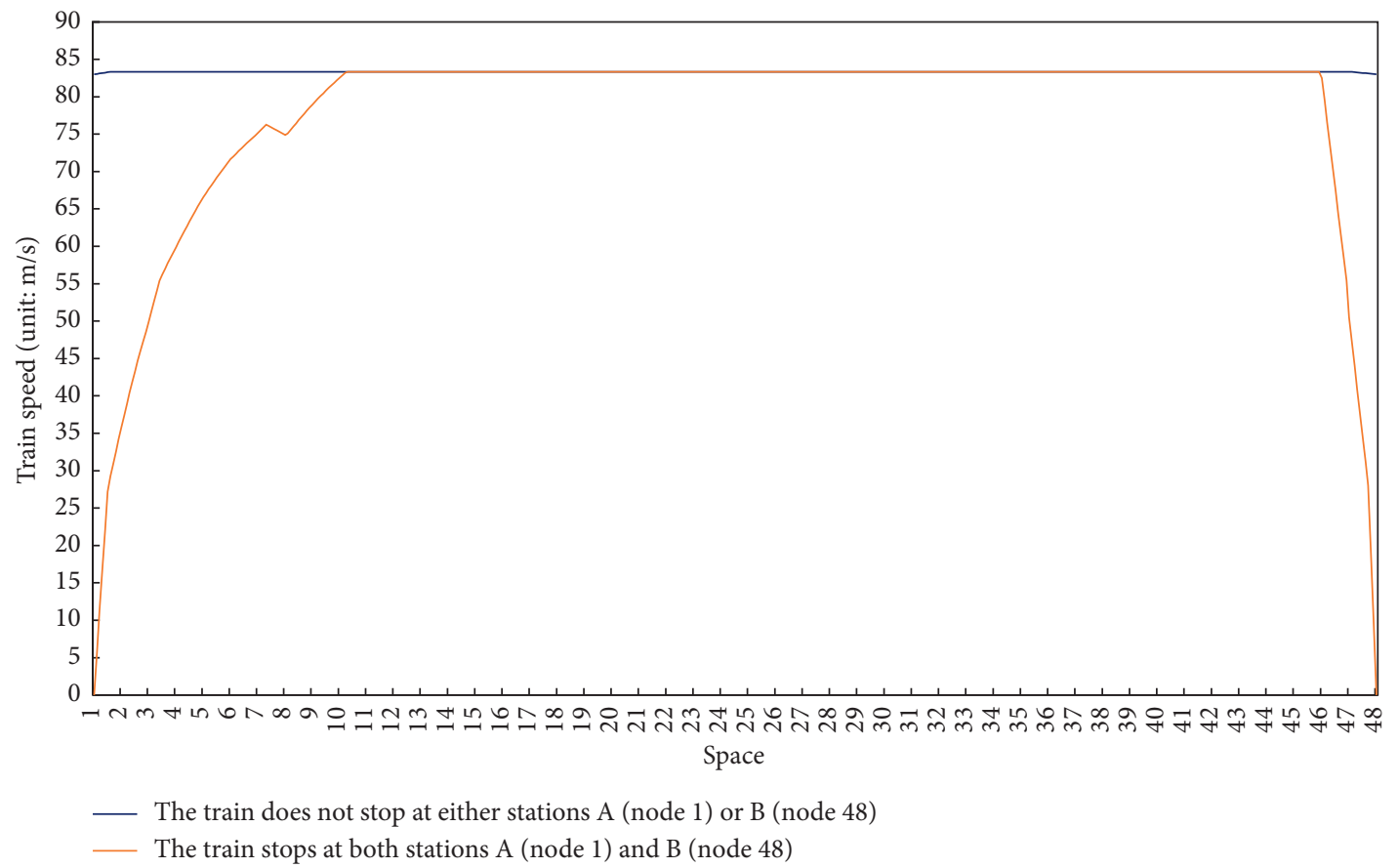

FIgURE 3: Train trajectories without a TSR.

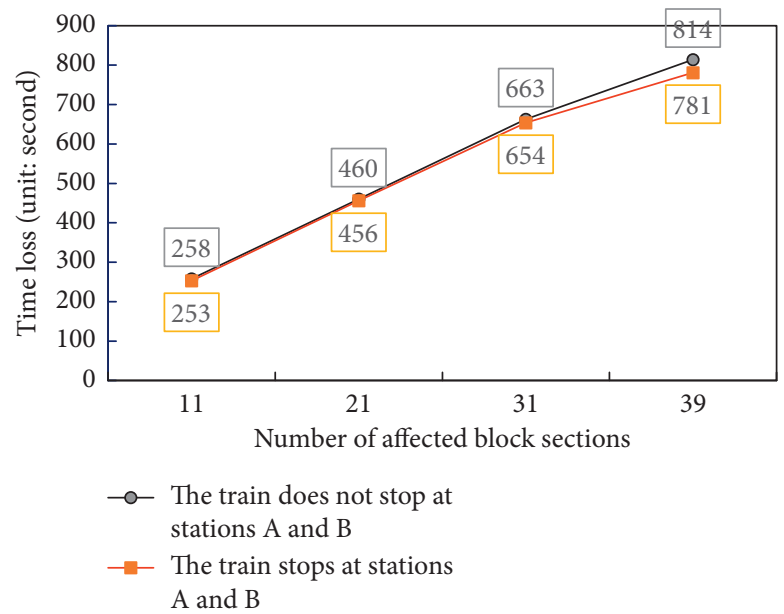

Figure 4: The time loss for different numbers of affected block sections.

4.2.2. Impact of the Location of the TSR. In this section, we study the impact of the location of the TSR that specifies the phases of acceleration, cruising, and deceleration. Without loss of generality, we use three sets of experiments with different values of the limited speed to analyse the impact. The time loss of each experiment is illustrated in Figure 7. The train trajectories of the three sets of cases are similar; thus, we report only the train trajectories of cases with a limited speed value of $44.44 \mathrm{~m} / \mathrm{s}$ in Figure 8 . It can be seen that if a TSR is in a location where the train is cruising, then it will yield more time loss (e.g., a 28\% increase in time loss at most) than a similar TSR located where the train accelerates or decelerates. Moreover, the time loss of a TSR located in the acceleration phase is less than that of a TSR located in the deceleration phase, attaining a $20 \%$ increase in time loss at most.

4.2.3. Impact of the Limited Speed Values. This section studies the influences of different severities of the TSR (i.e., distinguished by the limited speed value). The time loss under different limited speed values is shown in Figure 9, 


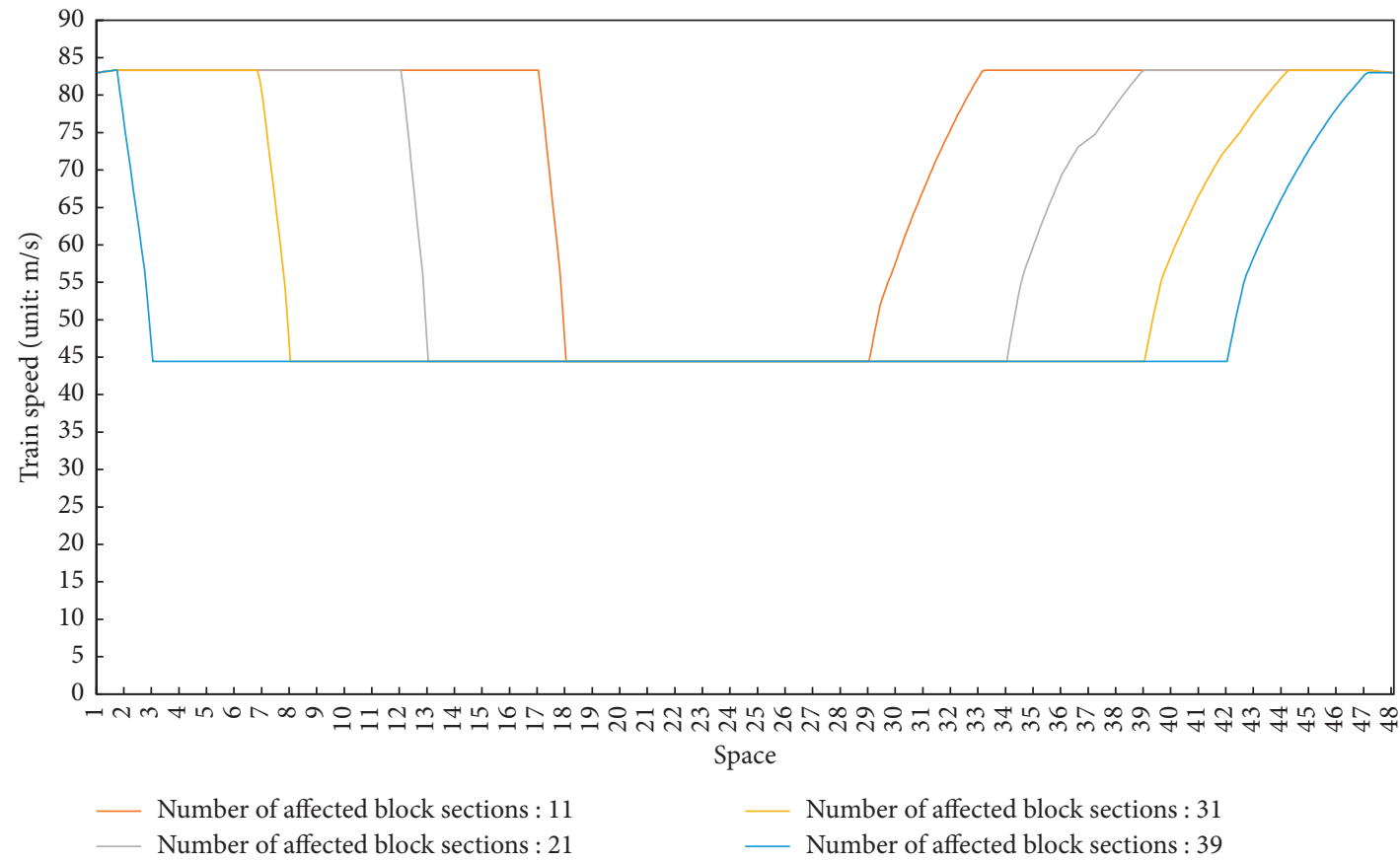

Figure 5: Train trajectories of a train that does not stop at either station A or B for different numbers of affected block sections.

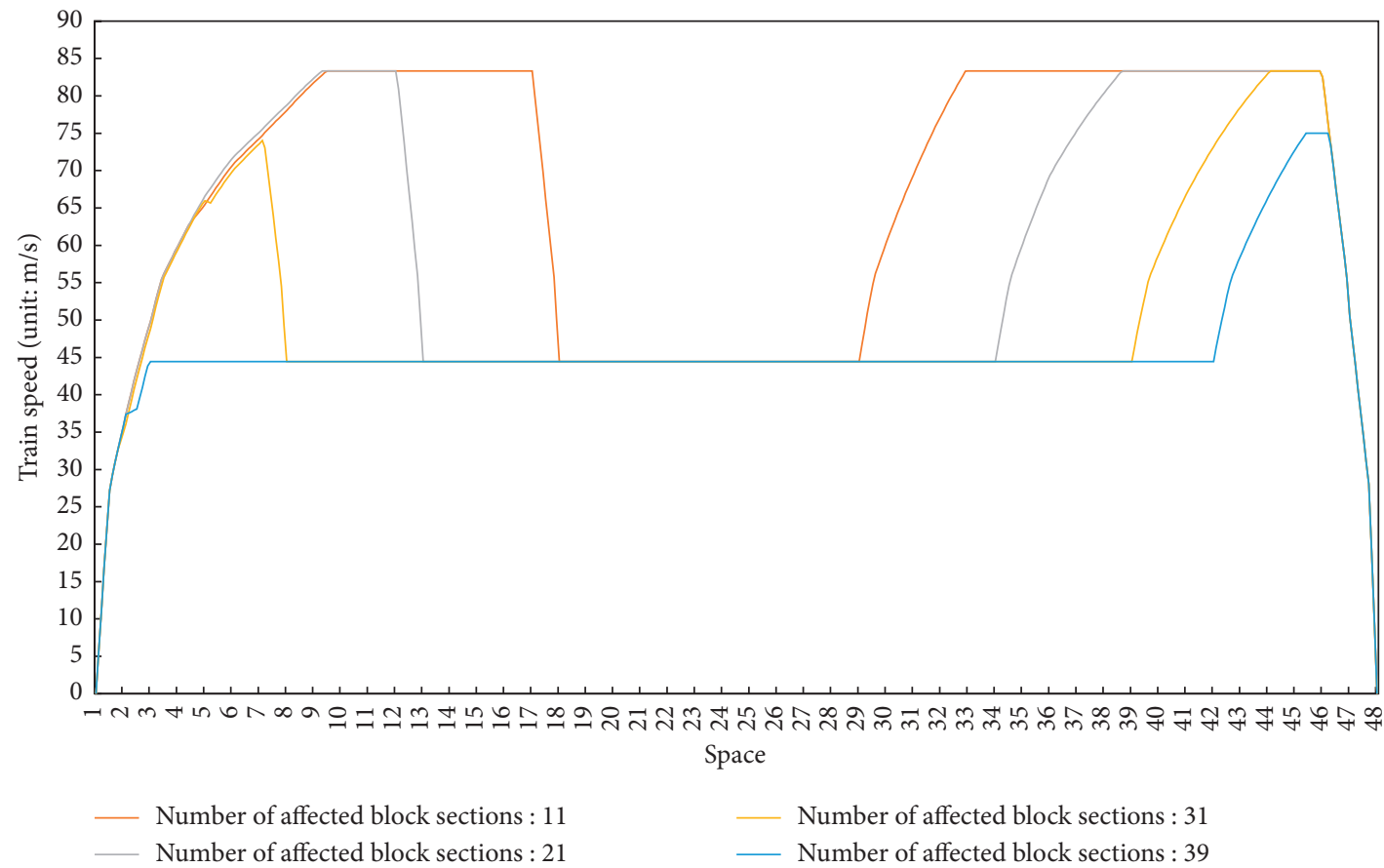

Figure 6: Train trajectories of a train that stops at both stations A and B for different numbers of affected block sections.

and the corresponding train trajectories are shown in Figure 10. It is easy to see that the time loss decreases with the increase in the limited speed value. The main time loss is due to the train running at the temporary lower speed. For example, the time loss at the lowest speed (i.e., $12.5 \mathrm{~m} / \mathrm{s}$ ) is $97 \%$ greater than that at the highest limited speed (i.e., $69.44 \mathrm{~m} / \mathrm{s})$.
4.2.4. Impact of the Time Duration of the TSR. We now study the impact of the time duration of a TSR. The time loss of each case is illustrated in Figure 11. The train trajectories under different cases are shown in Figure 12. It is obvious that the time loss increases with increasing time duration. Interestingly, in this study, when we continued to increase the time duration in excess of 30 minutes, the time loss did 


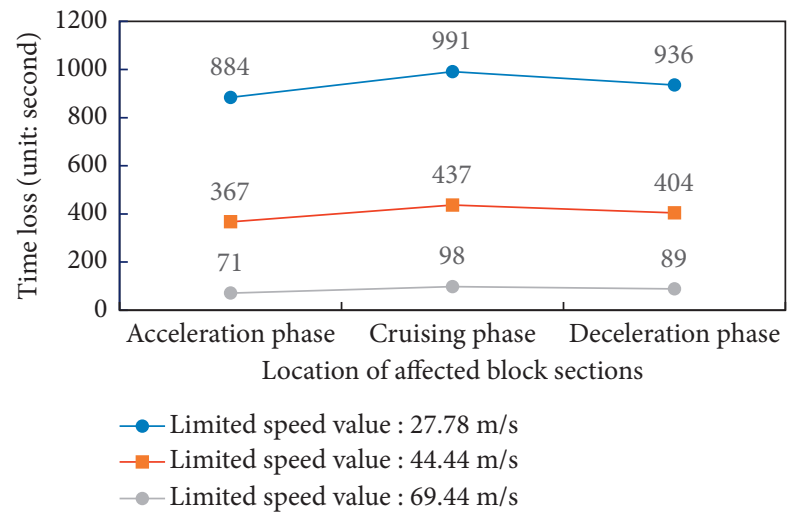

Figure 7: The time loss under different TSR locations.

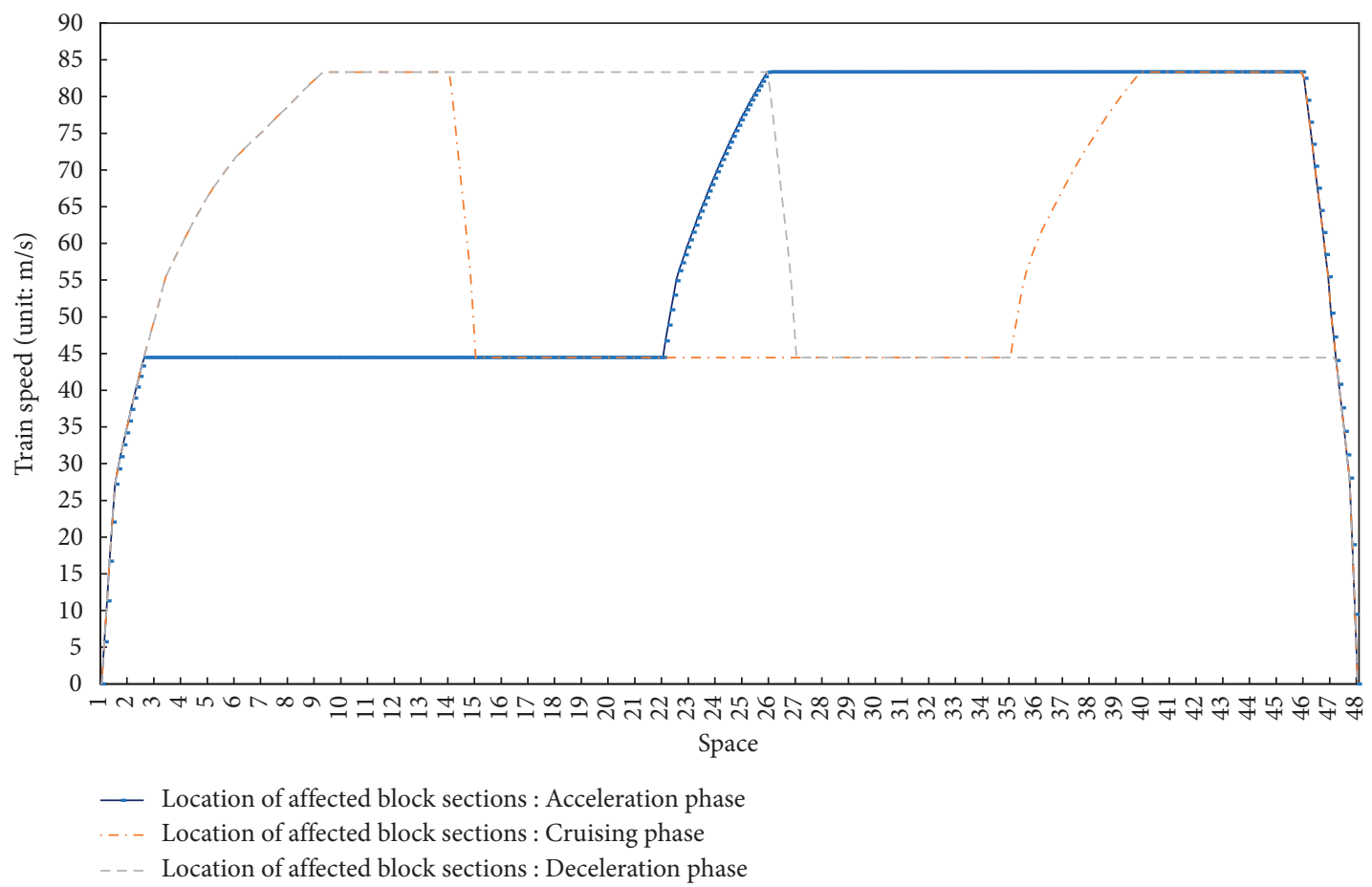

FIgURE 8: Train trajectories under different TSR locations for a limited speed value of $44.44 \mathrm{~m} / \mathrm{s}$.

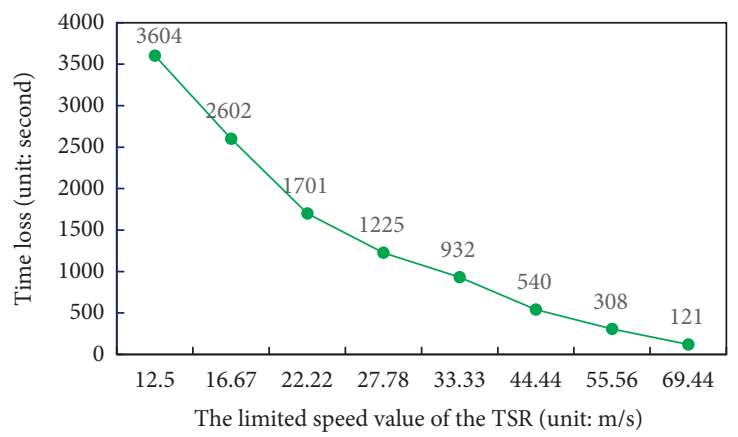

Figure 9: The time loss under different limited speed values. 


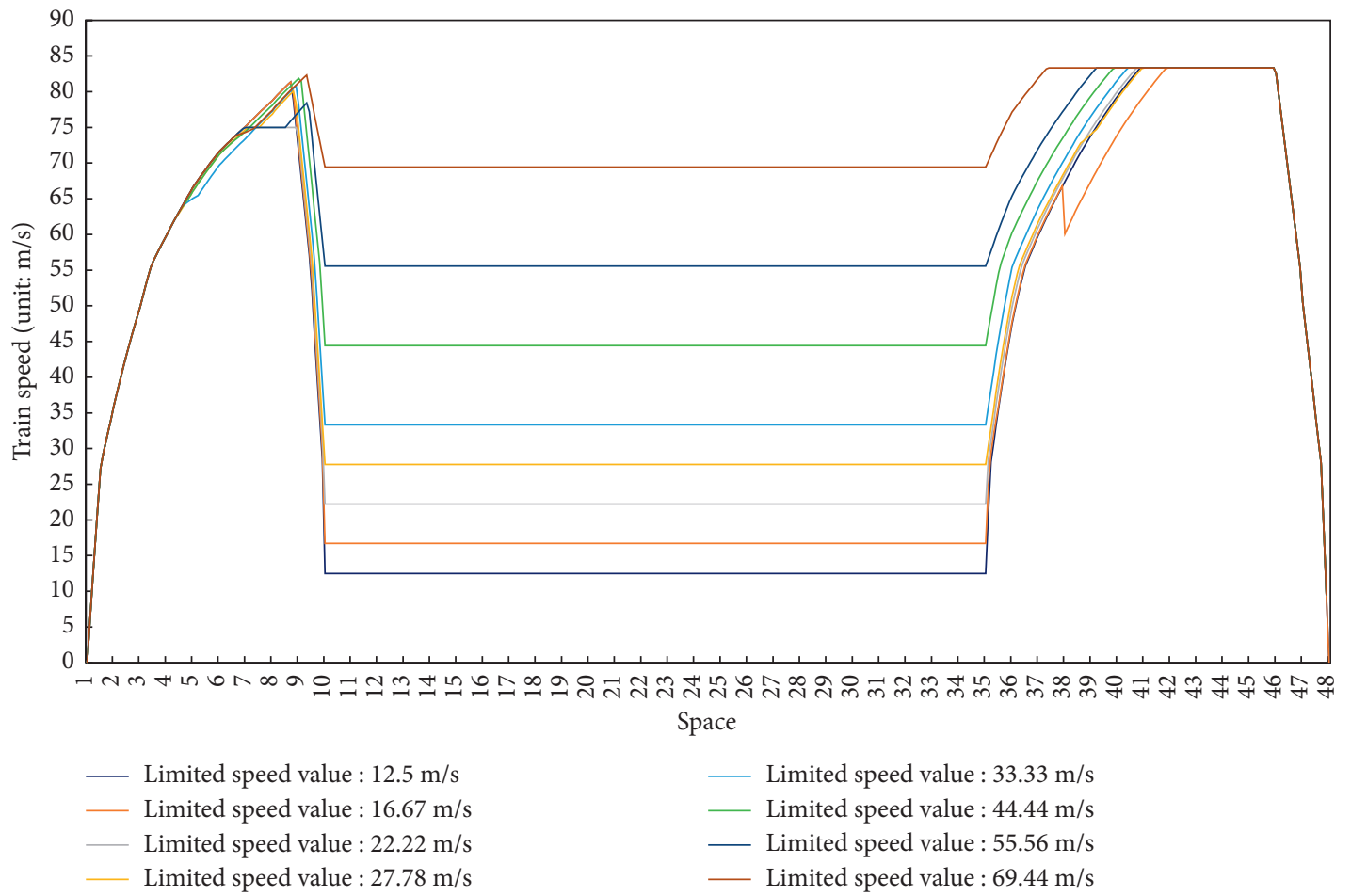

Figure 10: Train trajectories under different limited speed values.

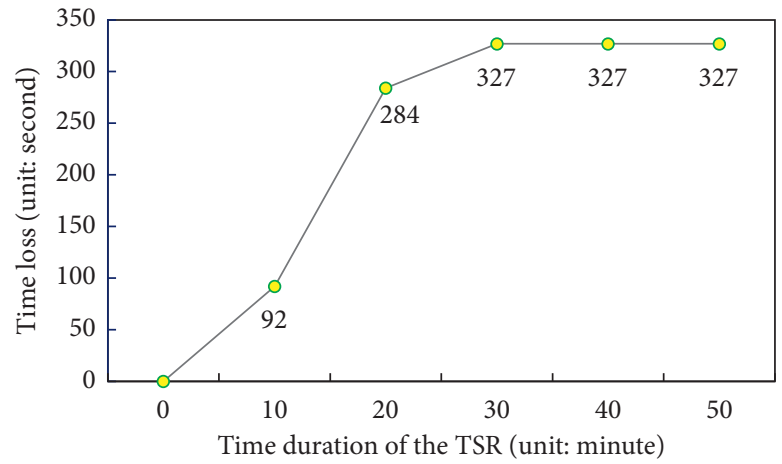

Figure 11: The time loss under different time durations.

not increase. This phenomenon may have occurred because the train left the TSR area within the 30 minute duration, as illustrated in Figure 12, where the train trajectories for time durations of 30,40 , and 50 minutes clearly coincide. That is, the train trajectory will not be influenced if the time duration is longer than 30 minutes, but the operations of subsequent trains and the high-speed railway line capacity will be affected, which need to be considered in future studies.

4.2.5. Impact of the Train Stopping Patterns. This section explores the impact of the train stopping patterns. As shown in Section 4.2.1, when the train stops at both stations, a $24 \%$ higher running time is needed than that when the train passes both stations due to the train acceleration and deceleration. Figure 4 reports the time loss of two stopping patterns under different numbers of affected block sections. The train running at a higher normal speed (i.e., the train that does not stop) has at most $4 \%$ more time loss than the train that stops. The reason is that if the train has an acceleration or deceleration phase, the train will run at a lower speed in the two phases, as shown in Section 4.2.2. Therefore, the train that stops will have a smaller difference between the normal speed and limited speed, further resulting in a lower time loss. 


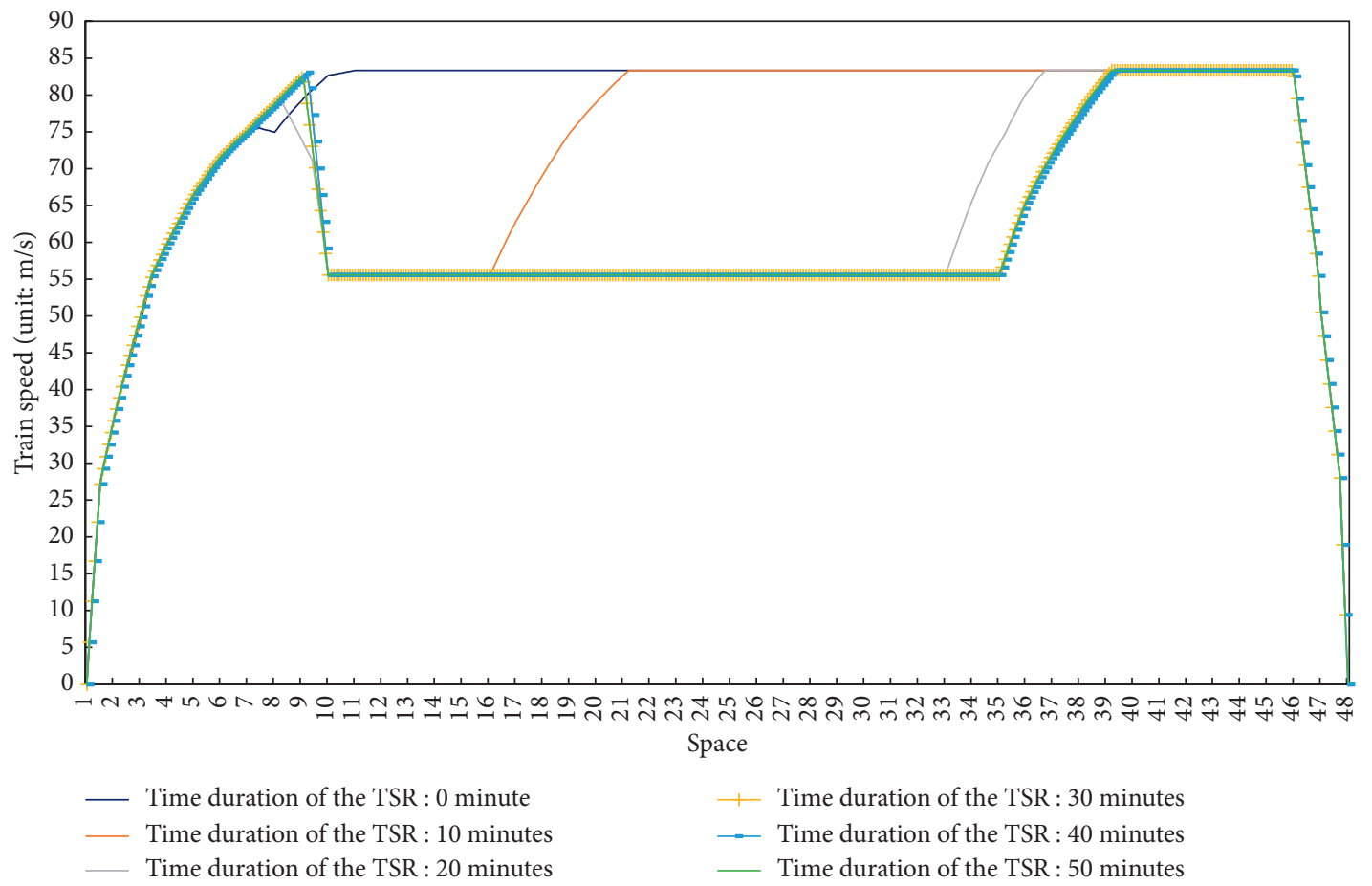

FIgURE 12: Train trajectories under different time durations.

\section{Conclusions}

In this paper, a discrete-space train movement model was constructed to evaluate the impact of a temporary speed restriction (TSR) on a high-speed railway train. The proposed model can determine whether a train will be affected by the block section influenced by the TSR within a certain time duration. Moreover, the model can calculate the train trajectories in detail and the train running time between two adjacent stations under different TSR conditions. The experiments evaluated the solution quality and computational efficiency of the proposed model. Moreover, a comprehensive analysis of the impact of several factors of the TSR was conducted, including the number of affected block sections, the location of the TSR, the limited speed value, and the stopping patterns of the train for two adjacent stations.

Our future research will focus on the following main extensions. First, it will be more helpful to provide dispatchers with detailed rescheduling plans for trains under TSR conditions. Thus, an integrated optimization model for train rescheduling and train control under a TSR needs to be studied. Second, it will be worth extending the discrete-time train movement model to determine whether a train will be affected by the block section influenced by the TSR within a time duration. A further step will then be to compare the performances of the proposed discrete-space train movement model and discrete-time train movement model, e.g., the measuring accuracy relative to the train running time and the influence of different TSR conditions on a train.

\section{Data Availability}

Previously reported data were used to support this study and are available in a book titled "Traction Calculation and Simulation System of EMU" by Ying Zhu, Xikui Lv, and Youding Xu (ASIN: B01CWZ2J0Y).

\section{Disclosure}

The authors are responsible for all results and opinions expressed in this paper.

\section{Conflicts of Interest}

The authors declare that they have no conflicts of interest.

\section{Acknowledgments}

This project was supported by the National Nature Science Foundation of China (61790573), State Key Laboratory of Rail Traffic Control and Safety, Beijing Jiaotong University (RCS2019ZJ001), Fundamental Research Funds for the Central Universities (2019YJS083), and China Railway Research and Development (K2018X012).

\section{References}

[1] P. Xu, F. Corman, and Q. Peng, "Analyzing railway disruptions and their impact on delayed traffic in Chinese highspeed railway," IFAC-PapersOnLine, vol. 49, no. 3, pp. 84-89, 2016. 
[2] J. Wang and D. Zhang, "Research on the transmission and execution of the train control system temporary speed restriction command," in Proceedings of the 2010 WASE International Conference on Information Engineering, pp. 344-347, Beidaihe, China, 2010.

[3] P. Xu, F. Corman, Q. Peng, and X. Luan, "A train rescheduling model integrating speed management during disruptions of high-speed traffic under a quasi-moving block system," Transportation Research Part B: Methodological, vol. 104, pp. 638-666, 2017.

[4] S. Long, L. Meng, Y. Wang et al., "Train rescheduling optimization model with considering the train control for a highspeed railway line under temporary speed restriction," in Proceedings of the The IEEE Intelligent Transportation Systems Conference ITSC 2019, Auckland, New Zealand, October 2019.

[5] X. Li, L. Meng, Y. Wang et al., "A rescheduling optimization model under temporary speed restriction," in Proceedings of the The IEEE Intelligent Transportation Systems Conference ITSC 2019, Auckland, New Zealand, October 2019.

[6] R. B. der Kooij, A. D. Landmark, A. A. Seim, and N. O. E. Olsson, "The effect of temporary speed restrictions, analyzed by using real train traffic data," Transportation Research Procedia, vol. 22, pp. 580-587, 2007.

[7] L. Yang, F. Li, Z. Gao, and K. Li, "Discrete-time movement model of a group of trains on a rail line with stochastic disturbance," Chinese Physics B, vol. 19, no. 10, Article ID 100510, 2010.

[8] Y.-H. Sun, C.-X. Cao, Y. Xu, and C. Wu, "Scheduling of highspeed rail traffic based on discrete-time movement model," Chinese Physics B, vol. 22, no. 12, p. 120501, 2013.

[9] R. Franke, P. Terwiesch, and M. Meyer, "An algorithm for the optimal control of the driving of trains," in Proceedings of the 39th IEEE Conference on Decision and Control, pp. 2123-2128, Sydney, Australia, December 2003.

[10] P. Howlett, "The optimal control of a train," Annals of Operations Research, vol. 98, no. 1-4, pp. 65-87, 2000.

[11] E. Khmelnitsky, "On an optimal control problem of train operation," IEEE Transactions on Automatic Control, vol. 45, no. 7, pp. 1257-1266, 2000.

[12] R. Liu and I. M. Golovitcher, "Energy-efficient operation of rail vehicles," Transportation Research Part A: Policy and Practice, vol. 37, no. 10, pp. 917-932, 2003.

[13] Y. Wang, Optimal Trajectory Planning and Train Scheduling for Railway Systems, Ph.D. thesis, Delft University of Technology, Delft, Netherlands, 2014.

[14] S. Lu, M. Q. Wang, P. Weston, S. Chen, and J. Yang, "Partial train speed trajectory optimization using mixed-integer linear programming," IEEE Transactions on Intelligent Transportation Systems, vol. 17, no. 10, pp. 2911-2920, 2016.

[15] Z. Tan, S. Lu, F. Xue et al., "A speed trajectory optimization model for rail vehicles using mixed integer linear programming," in Proceedings of the IEEE 20th International Conference on Intelligent Transportation Systems (ITSC), pp. 1-6, Yokohama, Japan, October 2017.

[16] C. Wu, W. Zhang, S. Lu, Z. Tan, F. Xue, and J. Yang, "Train speed trajectory optimization with on-board energy storage device," IEEE Transactions on Intelligent Transportation Systems, vol. 20, no. 11, pp. 4092-4102, 2019.

[17] I. A. Hansen and J. Pachl, Railway Timetable \& Traffic: Analysis, Modelling, Simulation, Eurail press, Utrecht, Netherlands, 1st edition, 2008. 